\title{
A mechanistic investigation of sustainable solvent-free, seed-directed synthesis of ZSM-5 zeolites in the absence of an organic structure-directing agent
}

Grandprix T. M. Kadja ${ }^{1,2,3 *}$, Noerma J. Azhari ${ }^{1}$, Rino R. Mukti ${ }^{1,2,3}$, Munawar Khalil ${ }^{1}$ Division of Inorganic and Physical Chemistry, Faculty of Mathematics and Natural Sciences, Institut Teknologi Bandung, Jalan Ganesha no. 10, Bandung 40132, Indonesia ${ }^{2}$ Center for Catalysis and Reaction Engineering, Institut Teknologi Bandung, Jalan Ganesha no. 10, Bandung 40132, Indonesia

${ }^{3}$ Research Center for Nanosciences and Nanotechnology, Institut Teknologi Bandung, Jalan Ganesha no. 10, Bandung 40132, Indonesia *Corresponding email: kadja@chem.itb.ac.id

\section{Synthesis of the seed}

The synthesis of the ZSM-5 seed was performed using the following prescursors, $\mathrm{Al}_{2}\left(\mathrm{SO}_{4}\right)_{3} .18 \mathrm{H}_{2} \mathrm{O}, \mathrm{Na}_{2} \mathrm{SiO}_{3} .9 \mathrm{H}_{2} \mathrm{O}, \mathrm{TPABr}, \mathrm{NH}_{4} \mathrm{Cl}$, and deionized water. They were mixed and grounded in an agate mortar for $20 \mathrm{~min}$ to form the mixture with a molar composition of 1 $\mathrm{SiO}_{2}$ : $0.91 \mathrm{NaOH}: 4.09 \mathrm{H}_{2} \mathrm{O}: 0.04 \mathrm{Al}_{2} \mathrm{O}_{3}: 0.74 \mathrm{NH}_{4} \mathrm{Cl}: 0.088 \mathrm{TPABr}$. The mixture was transferred into a Teflon-sealed autoclave and, subsequently placed in a preheated oven at 200 ${ }^{\circ} \mathrm{C}$ for $4.5 \mathrm{~h}$. After the crystallization, the products were filtered, washed, and dried at $80{ }^{\circ} \mathrm{C}$ overnight. The obtained white powder was calcined at $550{ }^{\circ} \mathrm{C}$ for $6 \mathrm{~h}$. 


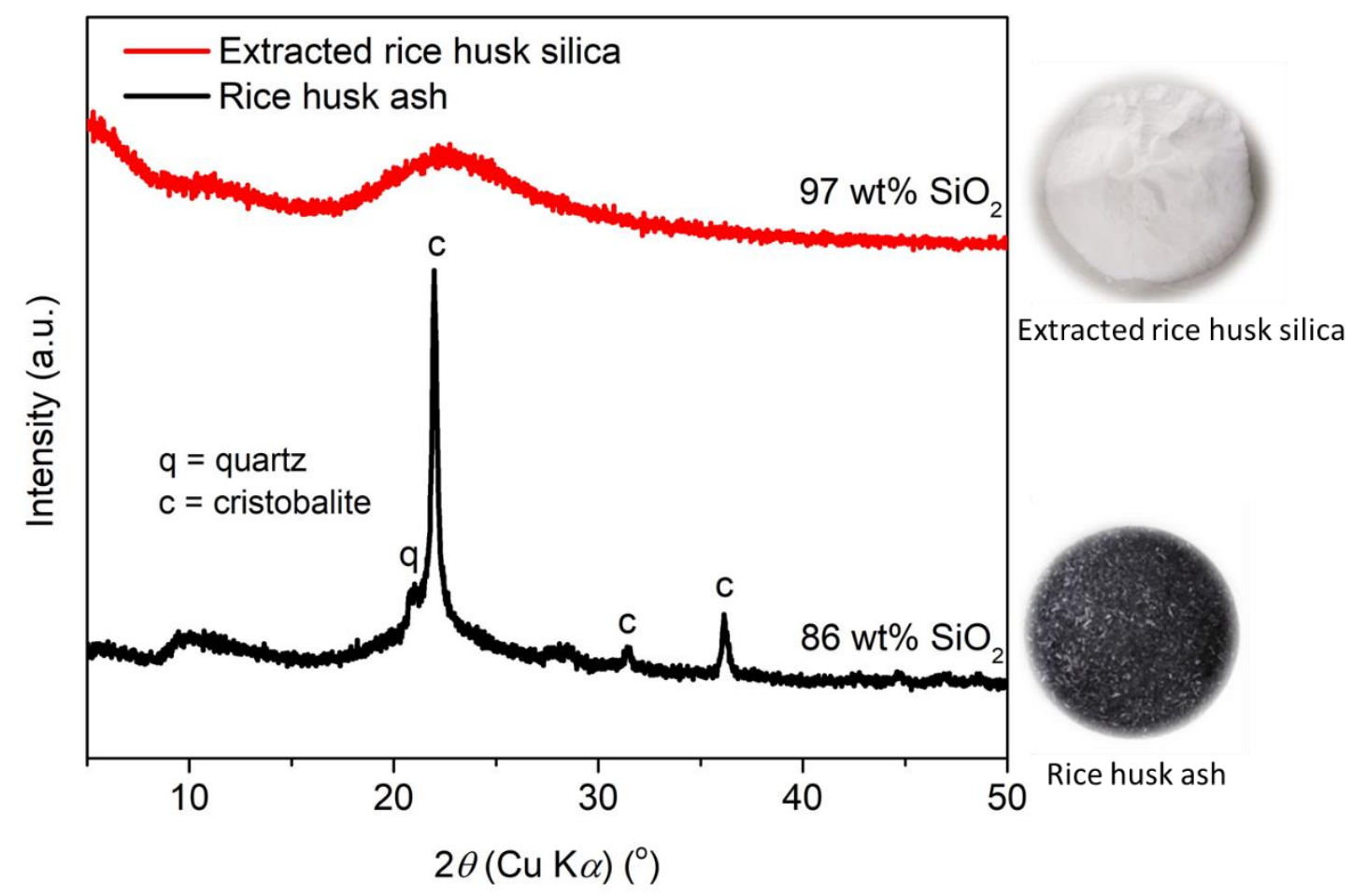

Figure S1. (left) XRD patterns of rice husk ash (RHA) and extracted silica from RHA and (right) their corresponding photographs. "Photograph courtesy of 'Noerma J. Azhari'. Copyright 2020." 


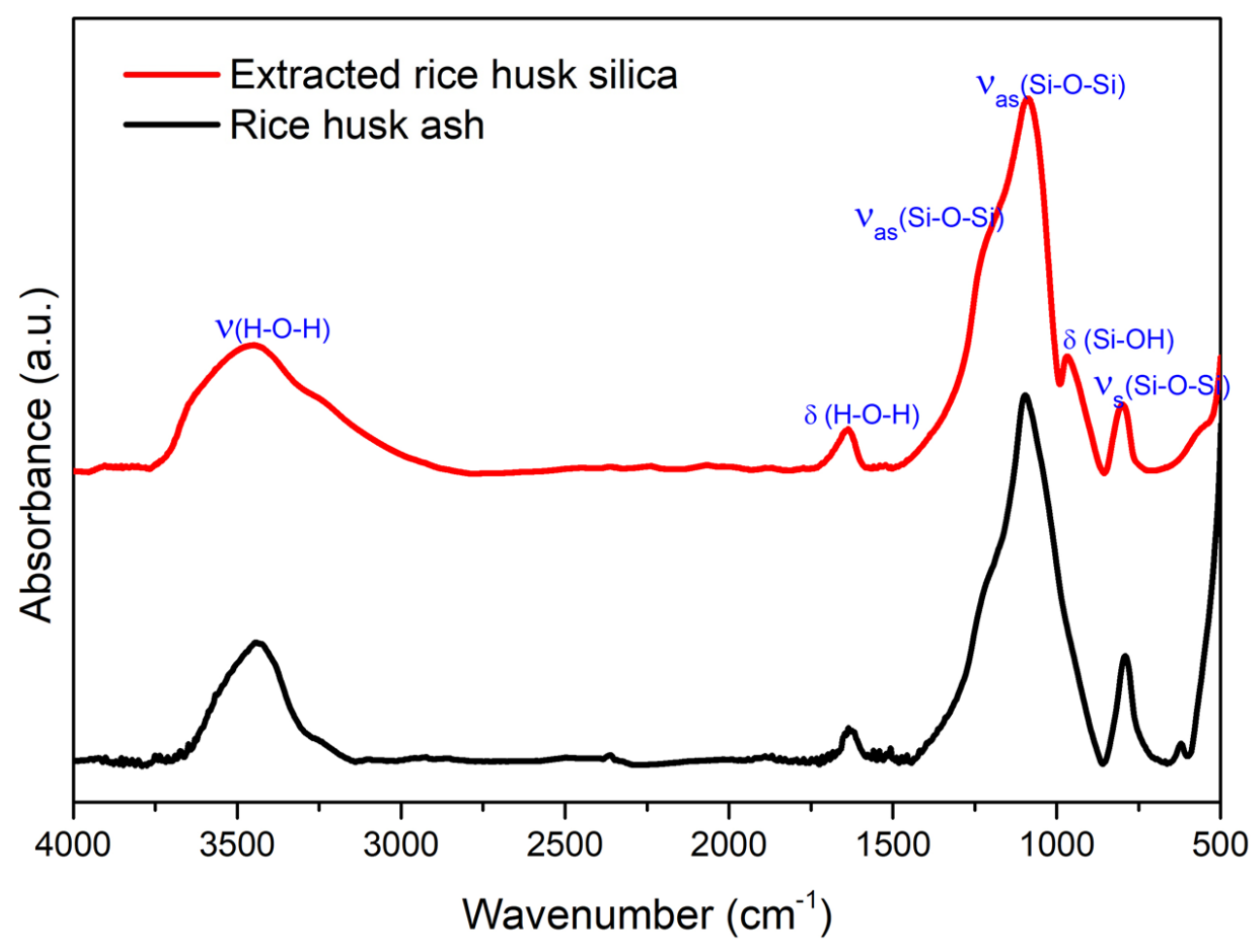

Figure S2. FTIR spectra of rice husk ash (RHA) and extracted silica from RHA.

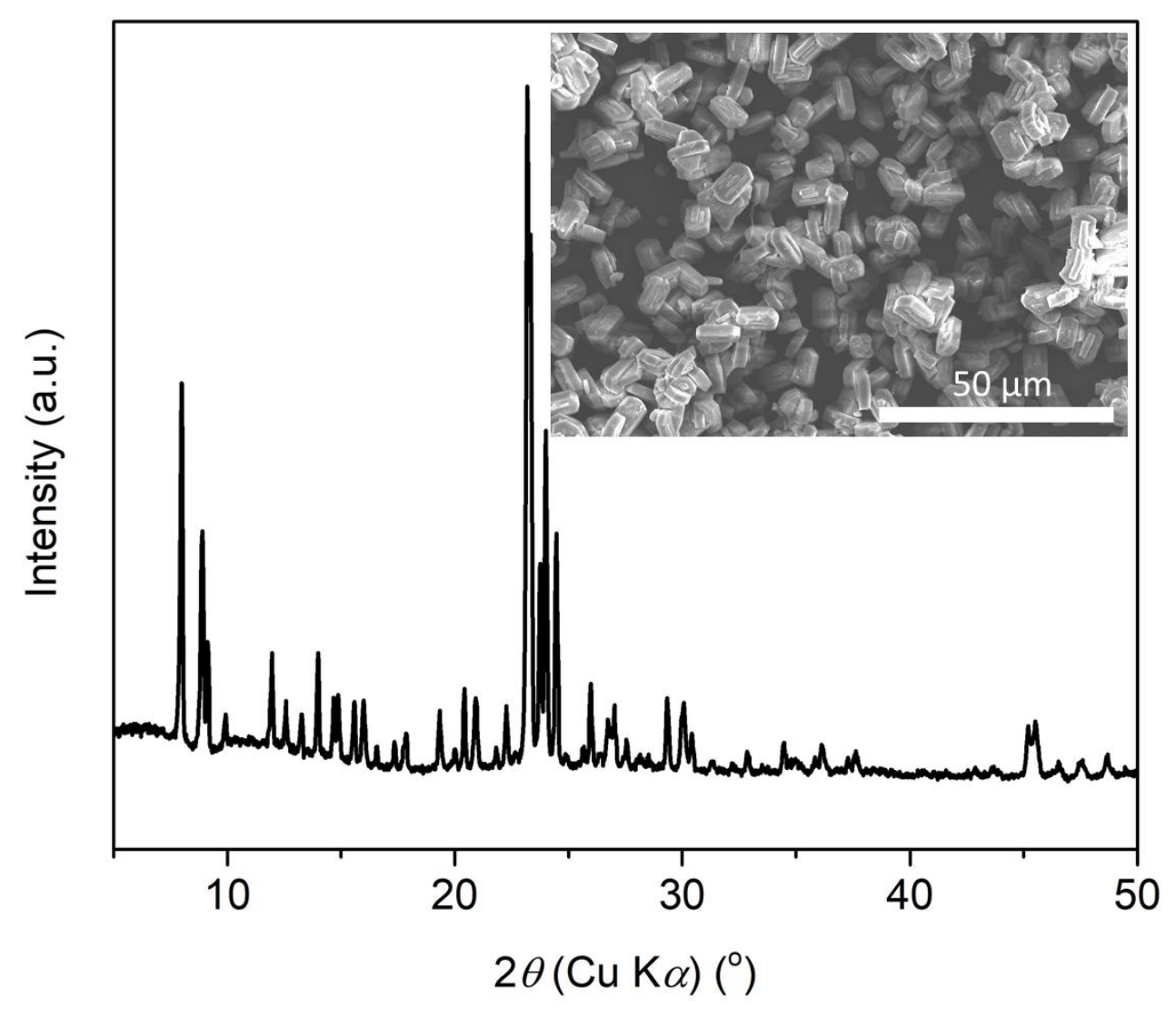

Figure S3. XRD pattern and SEM image of the seed. 
$4 \mathrm{~h}$

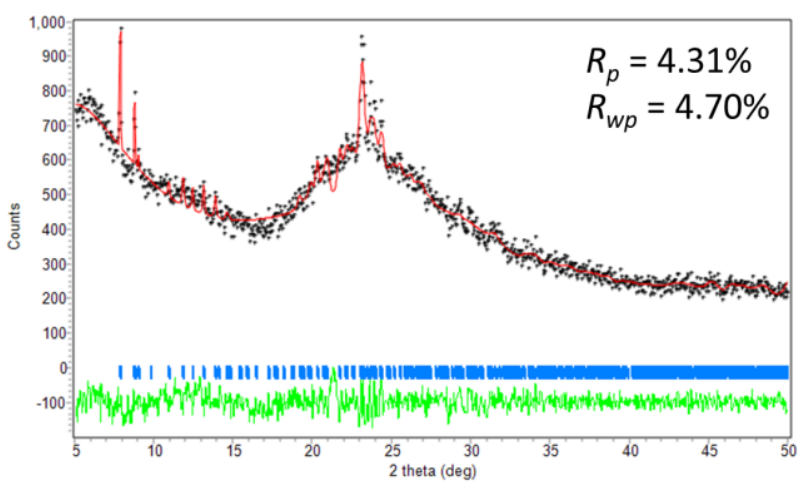

$5 \mathrm{~h}$

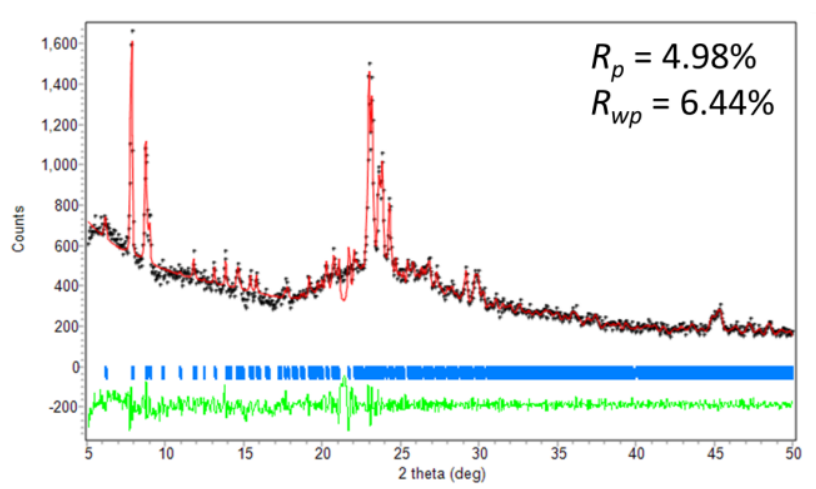

$6 \mathrm{~h}$

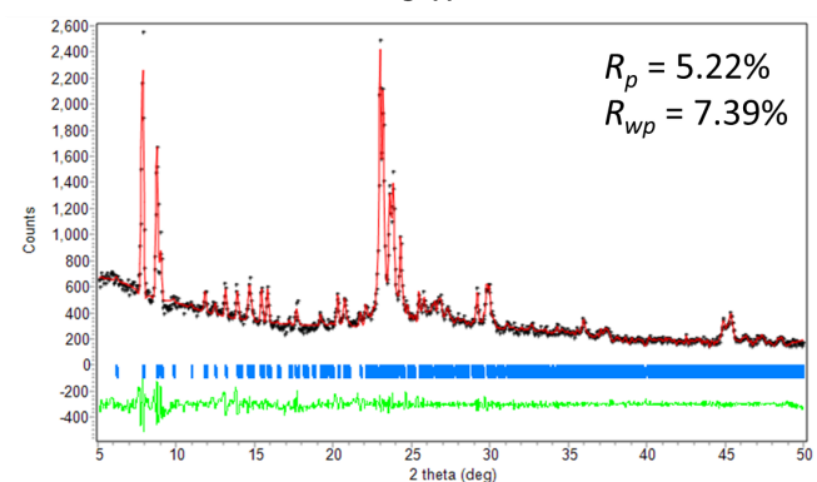

$10 \mathrm{~h}$
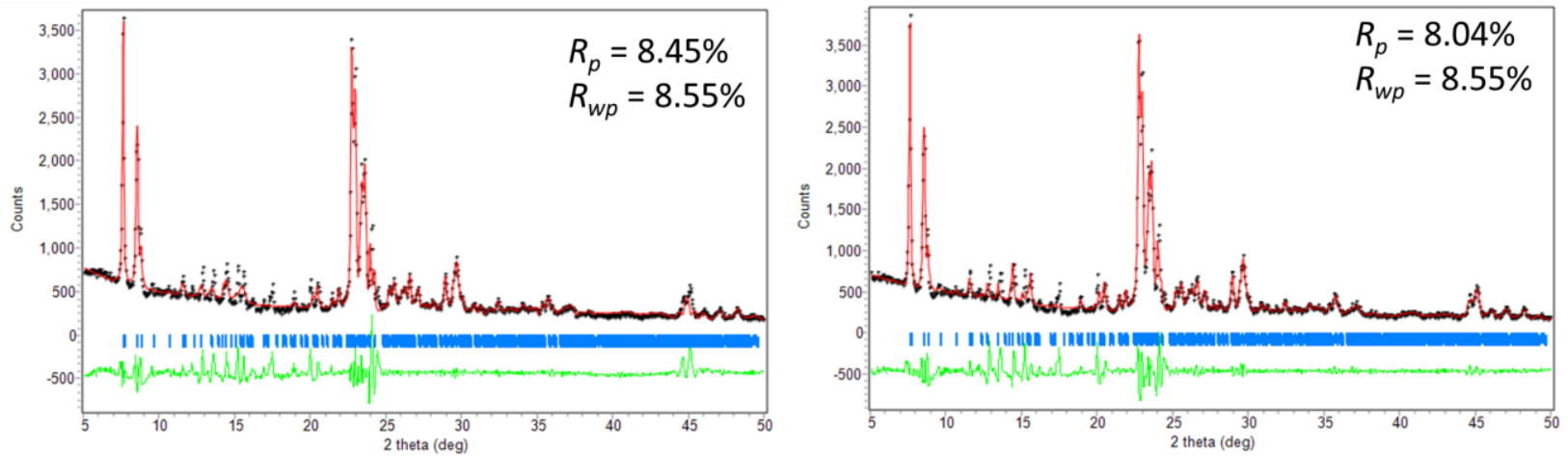

Figure S4. Whole pattern fitting using Le Bail method of XRD patterns of the products synthesized at $180{ }^{\circ} \mathrm{C}$. 


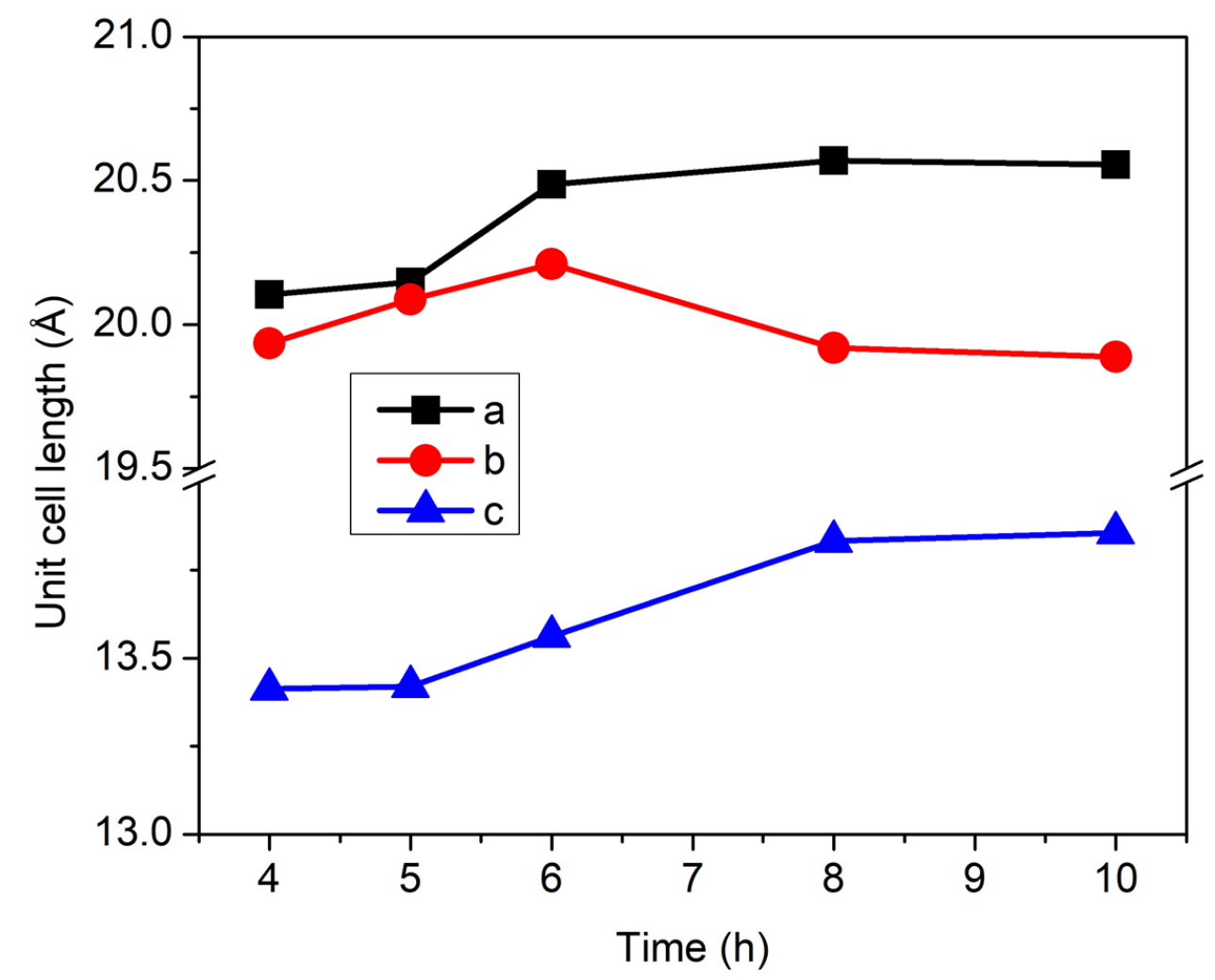

Figure S5. Lattice parameters of the products obtained after different periods

(a)

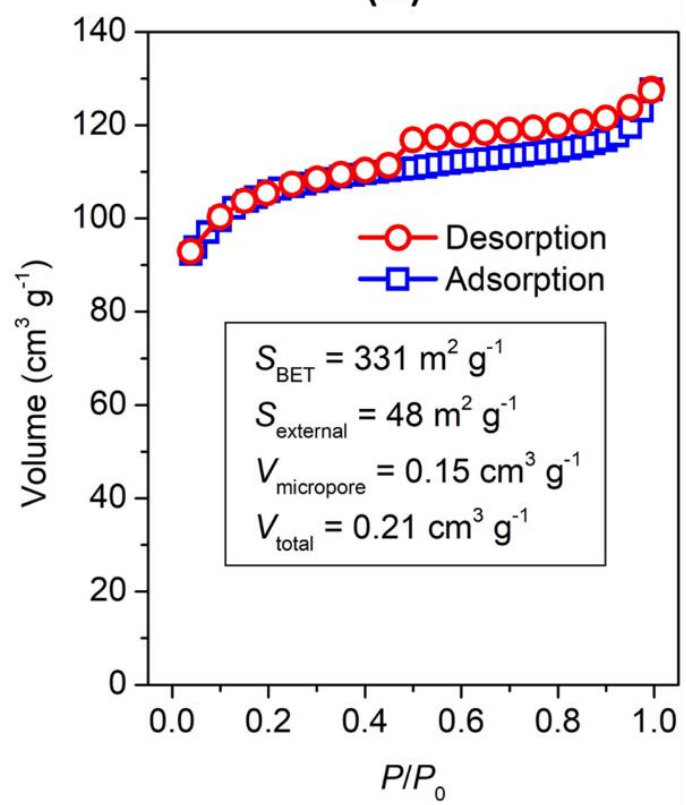

(b)

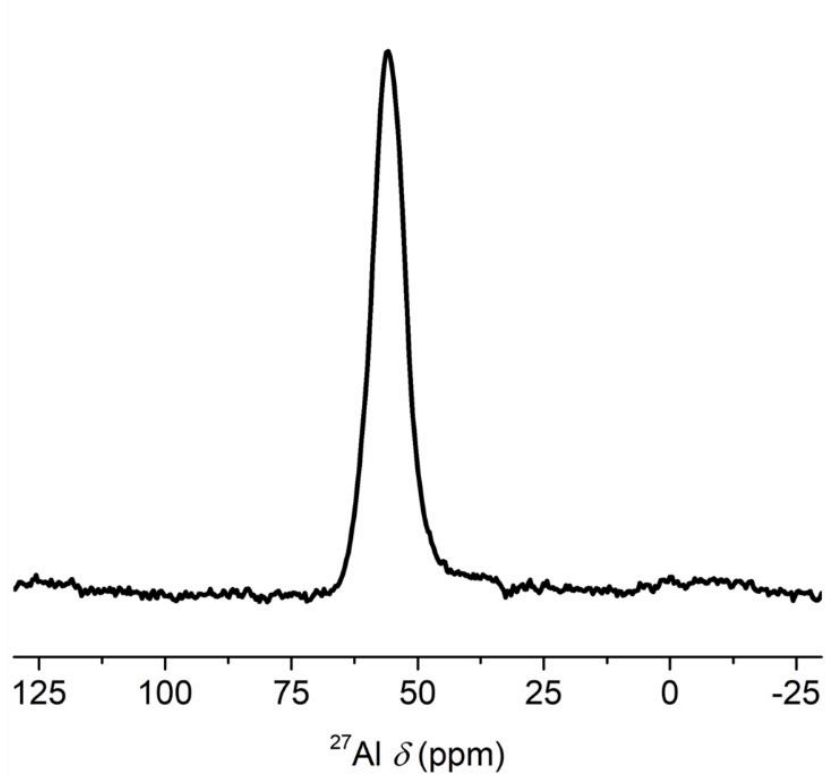

Figure S6. (a) $\mathrm{N}_{2}$ physisorption isotherm and (b) ${ }^{27} \mathrm{Al}$ MAS NMR spectroscopy of the products synthesized at $180{ }^{\circ} \mathrm{C}$ for $10 \mathrm{~h}$. 


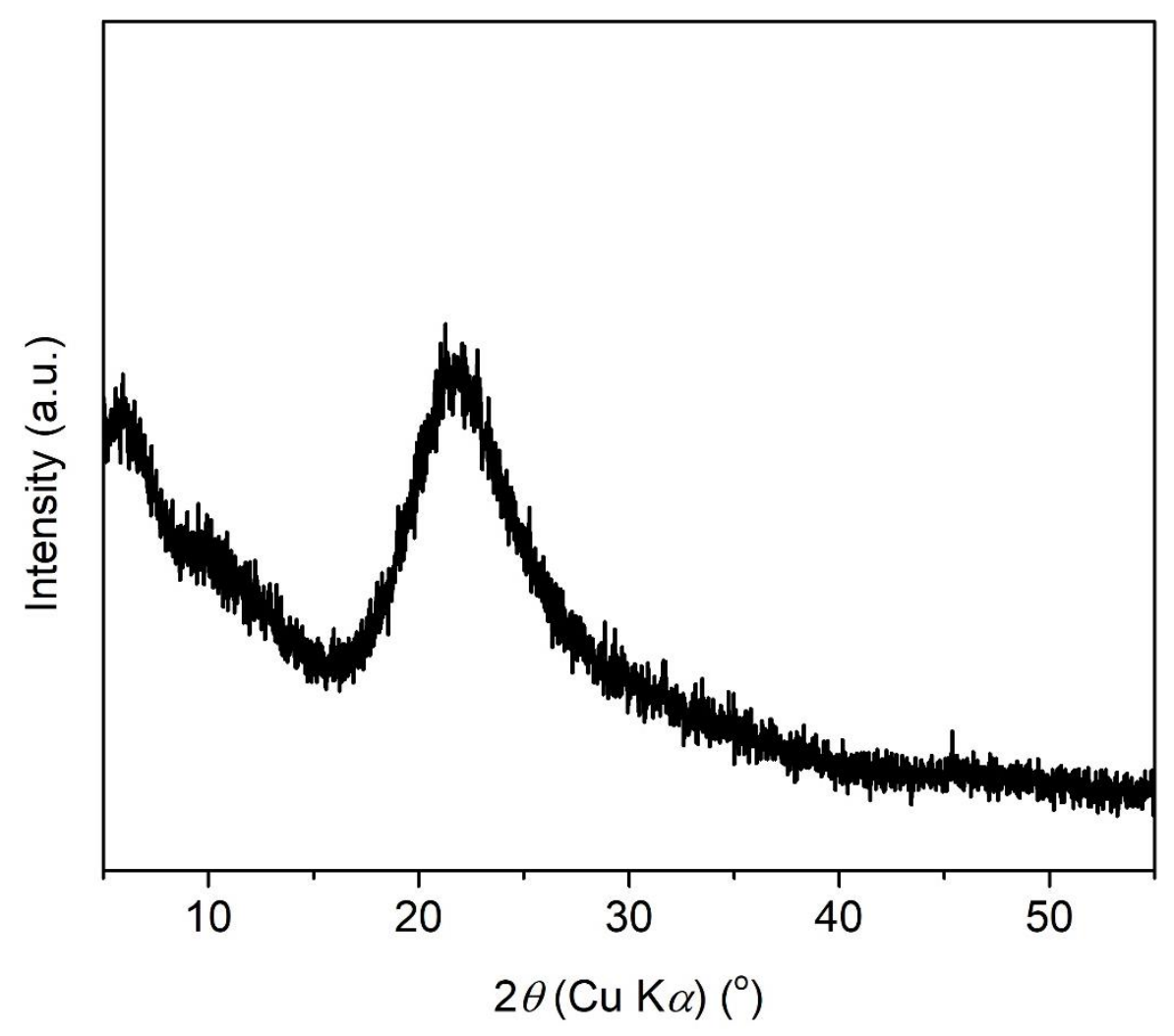

Figure S7. XRD pattern of the products crystallized at $180{ }^{\circ} \mathrm{C}$ for $10 \mathrm{~h}$ in the absence of seed.

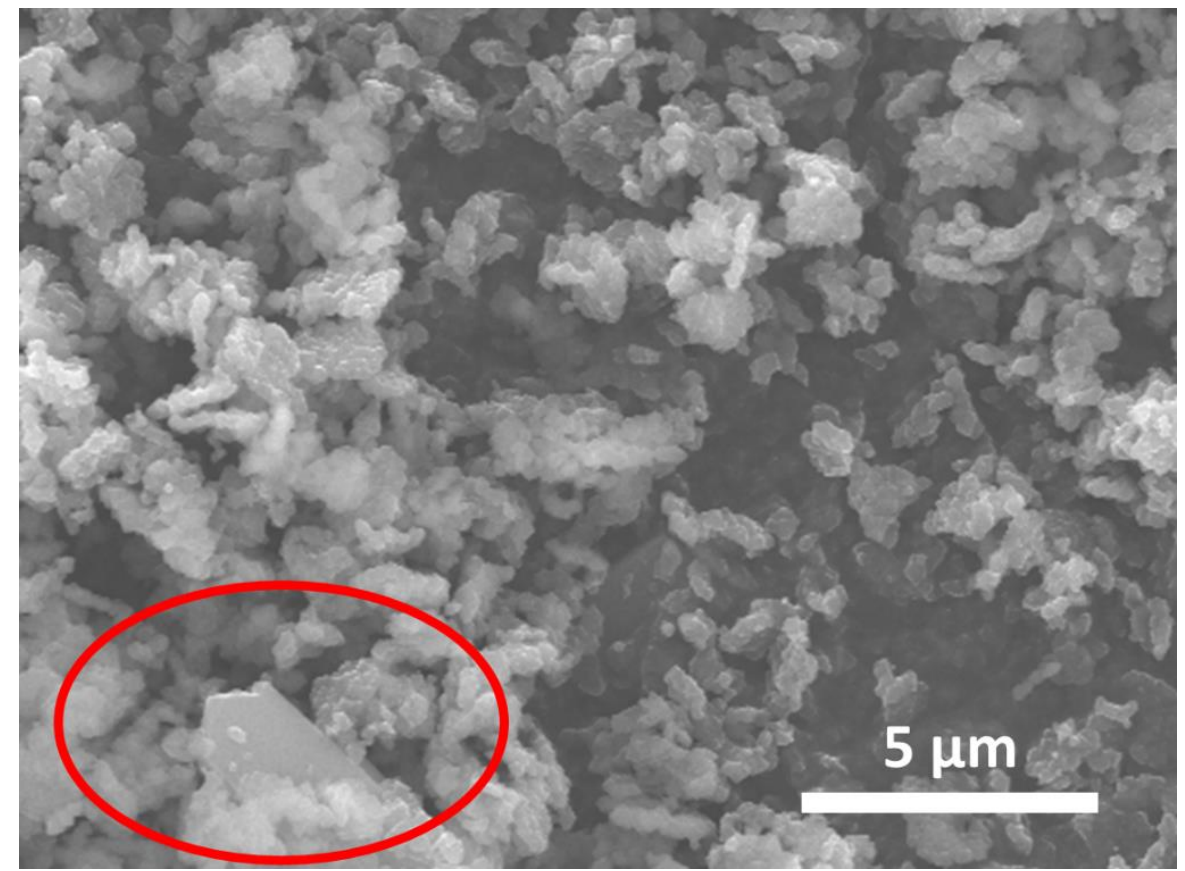

Figure S8. SEM image of the products crystallized at $180{ }^{\circ} \mathrm{C}$ for $10 \mathrm{~h}$. Red circle indicates the presence of the seed as the larger crystal. 
(a)

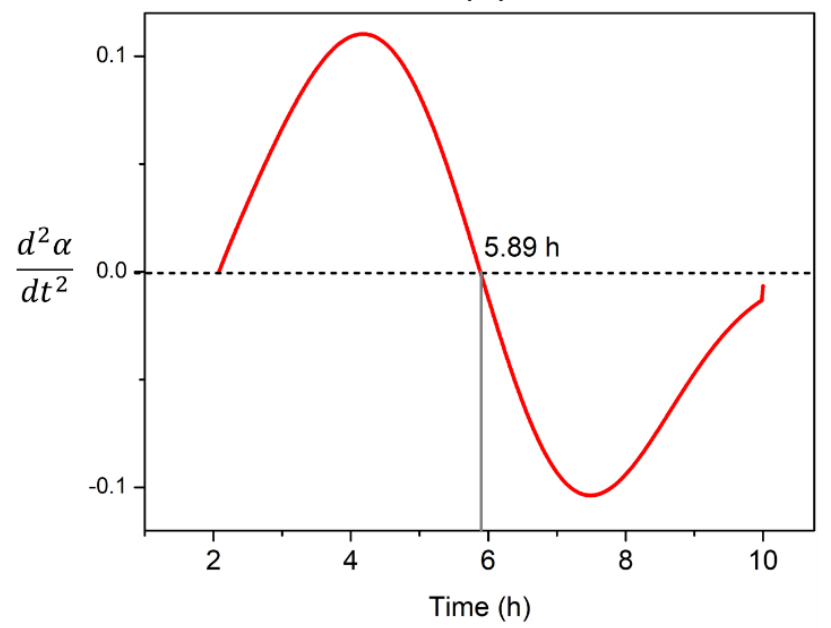

(b)

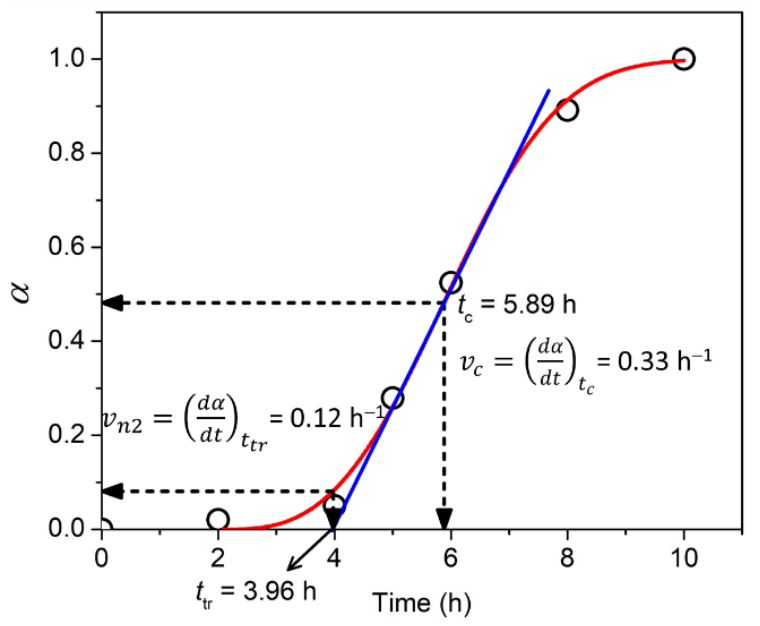

Figure S9. (a) Second derivative of the crystallization curve, and (b) scheme for determining transition and crystal growth stages at $180{ }^{\circ} \mathrm{C}$. 
Table S1. Experimental condition, kinetic parameters, and activation energy data from literature. all was performed under hydrothermal method.

\begin{tabular}{|c|c|c|c|c|c|c|c|c|c|c|c|c|}
\hline \multirow[t]{2}{*}{ Composition } & \multirow{2}{*}{$\begin{array}{l}\text { Silica } \\
\text { source }\end{array}$} & \multirow{2}{*}{$\begin{array}{l}\text { Temper } \\
\text { ature } \\
\left(\begin{array}{ll} & \\
& \mathrm{C}\end{array}\right)\end{array}$} & \multirow{2}{*}{$\begin{array}{l}\text { Use or } \\
\text { not of } \\
\text { OSDA }\end{array}$} & \multicolumn{5}{|c|}{ Kinetic parameters } & \multicolumn{3}{|c|}{$\begin{array}{l}\text { Energy activation } \\
(\mathrm{kJ} / \mathrm{mol})\end{array}$} & \multirow[t]{2}{*}{ Ref. } \\
\hline & & & & $t_{0}(h)$ & $\mathrm{t}_{\mathrm{tr}}(\mathrm{h})$ & $t_{c}(h)$ & $\mathrm{k}$ & $\mathrm{n}$ & $E_{n 1}$ & $E_{n 2}$ & $\mathrm{E}_{\mathrm{c}}$ & \\
\hline \multirow{3}{*}{$\begin{array}{l}40 \mathrm{SiO}_{2}: \mathrm{Al}_{2} \mathrm{O}_{3}: 0.38 \mathrm{Na}_{2} \\
\mathrm{O}: 45 \mathrm{H}_{2} \mathrm{O}\end{array}$} & \multirow[t]{3}{*}{ perlite } & 170 & \multirow{3}{*}{$\begin{array}{c}\text { Seed } \\
\text { assisted } \\
(6 \%)\end{array}$} & 12.2 & 21.7 & 28.3 & $3.41 \times 10^{-5}$ & 3.5 & \multirow[t]{3}{*}{60} & \multirow[t]{3}{*}{64} & \multirow[t]{3}{*}{48} & \multirow[t]{3}{*}{1} \\
\hline & & 180 & & 7.0 & 13.4 & 18.4 & $2.02 \times 10^{-4}$ & 3.4 & & & & \\
\hline & & 190 & & 6.0 & 10.2 & 13.2 & $7.16 \times 10^{-4}$ & 3.5 & & & & \\
\hline \multirow{3}{*}{$\begin{array}{l}1 \mathrm{SiO}_{2}: 0.21 \mathrm{NaOH} \\
35.5 \mathrm{H}_{2} \mathrm{O}\end{array}$} & \multirow[t]{3}{*}{ kaolinite } & 170 & \multirow{3}{*}{$\begin{array}{l}\text { OSDA- } \\
\text { free }\end{array}$} & 15 & - & - & $1.18 \times 10^{-2}$ & 1.5 & \multirow[t]{3}{*}{78.31} & \multirow[t]{3}{*}{-} & \multirow[t]{3}{*}{68.53} & \multirow[t]{3}{*}{2} \\
\hline & & 180 & & 9 & - & - & $3.35 \times 10^{-2}$ & 1.5 & & & & \\
\hline & & 190 & & 6 & - & - & $2.26 \times 10^{-2}$ & 1.5 & & & & \\
\hline \multirow{3}{*}{$\begin{array}{l}30 \mathrm{Na}_{2} \mathrm{O}: 68(\mathrm{TPA})_{2} \mathrm{O}: \\
\mathrm{Al}_{2} \mathrm{O}_{3}: 111 \mathrm{SiO}_{2}: 4000 \\
\mathrm{H}_{2} \mathrm{O}: 25 \mathrm{H}_{2} \mathrm{SO}_{4}\end{array}$} & \multirow{3}{*}{$\begin{array}{c}\mathrm{Na}_{2} \mathrm{SiO}_{3} \\
\mathrm{nH}_{2} \mathrm{O}\end{array}$} & 150 & \multirow[t]{3}{*}{$\mathrm{TPABr}$} & - & - & 15.5 & - & \multirow[t]{3}{*}{4} & \multirow[t]{3}{*}{35} & \multirow[t]{3}{*}{62} & \multirow[t]{3}{*}{166} & \multirow[t]{3}{*}{3} \\
\hline & & 165 & & - & - & 12 & - & & & & & \\
\hline & & 180 & & - & - & 8 & - & & & & & \\
\hline \multirow{3}{*}{$\begin{array}{l}30 \mathrm{Na}_{2} \mathrm{O}: 68(\mathrm{py})_{2} \mathrm{O}: \\
\mathrm{Al}_{2} \mathrm{O}_{3}: 111 \mathrm{SiO}_{2}: 4000 \\
\mathrm{H}_{2} \mathrm{O}: 25 \mathrm{H}_{2} \mathrm{SO}_{4}\end{array}$} & \multirow{3}{*}{$\begin{array}{c}\mathrm{Na}_{2} \mathrm{SiO}_{3} \\
\mathrm{nH}_{2} \mathrm{O}\end{array}$} & 150 & pyridine & - & - & 38.5 & - & 8 & 34 & 78 & 291 & 3 \\
\hline & & 165 & & - & - & 22 & - & & & & & \\
\hline & & 180 & & - & - & 18.5 & - & & & & & \\
\hline $14 \mathrm{Na}_{2} \mathrm{O}: 100 \mathrm{SiO}_{2^{-}}$ & Ludox & 150 & OSDA- & 18 & - & - & $2.21 \times 10^{-4}$ & 3.23 & 72.8 & 92.7 & 53.1 & 4 \\
\hline $1.5 \mathrm{Al}_{2} \mathrm{O}_{3}: 2500 \mathrm{H}_{2} \mathrm{O}$ & As- 40 & 170 & free & 8 & - & - & $2.99 \times 10^{-4}$ & 3.64 & & & & \\
\hline & & 190 & & 3 & - & - & $2.79 \times 10^{-3}$ & 3.39 & & & & \\
\hline $14 \mathrm{Na}_{2} \mathrm{O}: 100 \mathrm{SiO}_{2}$ & Ludox & 150 & OSDa- & 35 & - & - & $1.42 \times 10^{-5}$ & 3.60 & 99.5 & 125.9 & 88.7 & \\
\hline $2 \mathrm{Al}_{2} \mathrm{O}_{3}: 2500 \mathrm{H}_{2} \mathrm{O}$ & As -40 & 170 & free & 14.5 & - & - & $1.33 \times 10^{-4}$ & 4.21 & & & & \\
\hline & & 190 & & 3 & - & - & $2.29 \times 10^{-3}$ & 4.18 & & & & \\
\hline $14 \mathrm{Na}_{2} \mathrm{O}: 100 \mathrm{SiO}_{2}$ & Ludox & 150 & & 33 & - & - & $2.3 \times 10^{-4}$ & 3.0 & 113.6 & 120.0 & 80.8 & \\
\hline $3 \mathrm{Al}_{2} \mathrm{O}_{3}: 2500 \mathrm{H}_{2} \mathrm{O}$ & As-40 & 170 & & 12 & - & - & $4.9 \times 10^{-4}$ & 3.6 & & & & \\
\hline & & 190 & & 2 & - & - & $2.5 \times 10^{-3}$ & 4.2 & & & & \\
\hline
\end{tabular}




\begin{tabular}{|c|c|c|c|c|c|c|c|c|c|c|c|c|}
\hline \multirow{3}{*}{$\begin{array}{l}4.38 \text { (TPA })_{2} \mathrm{O}: 27.6 \mathrm{Na}_{2} \mathrm{O}: \\
\mathrm{A}_{2} \mathrm{O}_{3}: 87.7 \mathrm{SIO}_{2}: 3262 \\
\mathrm{H}_{2} \mathrm{O}\end{array}$} & \multirow{3}{*}{$\begin{array}{l}\text { Sodium } \\
\text { silicate }\end{array}$} & 160 & \multirow[t]{3}{*}{$\mathrm{TPABr}$} & - & - & - & $9.0 \times 10-2$ & 2.4 & \multirow[t]{3}{*}{199.5} & \multirow[t]{3}{*}{-} & \multirow[t]{3}{*}{238.6} & \multirow[t]{3}{*}{5} \\
\hline & & 180 & & - & - & - & $3,83 \times 10-1$ & 2.0 & & & & \\
\hline & & 200 & & - & - & - & $4.49 \times 10-1$ & 1.2 & & & & \\
\hline \multirow{2}{*}{$\begin{array}{l}4.38(\text { TEPA })_{2} \mathrm{O}: 27.6 \\
\mathrm{Na}_{2} \mathrm{O}: \mathrm{A}_{2} \mathrm{O}_{3}: 87.7 \mathrm{SIO}_{2}: \\
3262 \mathrm{H}_{2} \mathrm{O}\end{array}$} & \multirow{2}{*}{$\begin{array}{l}\text { Sodium } \\
\text { silicate }\end{array}$} & 180 & \multirow{2}{*}{ TEPABr } & - & - & - & $3.7 \times 10-2$ & 3.2 & \multirow{2}{*}{270.4} & \multirow{2}{*}{-} & \multirow{2}{*}{206.8} & \multirow{2}{*}{5} \\
\hline & & 200 & & - & - & - & $1.49 \times 10-1$ & 2.4 & & & & \\
\hline \multirow{2}{*}{$\begin{array}{l}3.36\left(\mathrm{TEBA}^{+}\right)_{2} \mathrm{O}: \\
25.4 \mathrm{Na}_{2} \mathrm{O}: \mathrm{A}_{1}{ }_{2} \mathrm{O}_{3}: 85 \\
\mathrm{SiO}_{2}: 3200 \mathrm{H}_{2} \mathrm{O}\end{array}$} & \multirow{2}{*}{$\begin{array}{l}\text { Sodium } \\
\text { silicate }\end{array}$} & 180 & \multirow{2}{*}{ TEBABr } & - & - & - & $4.0 \times 10-2$ & 4.5 & \multirow{2}{*}{118.1} & \multirow{2}{*}{-} & \multirow{2}{*}{78.6} & \multirow{2}{*}{6} \\
\hline & & 200 & & - & - & - & $8.2 \times 10-2$ & 2.0 & & & & \\
\hline \multirow{3}{*}{$\begin{array}{l}\text { 3.36(TEPA) }{ }_{2} \mathrm{O}: \\
25.4 \mathrm{Na}_{2} \mathrm{O}: \mathrm{A}_{1} \mathrm{O}_{3}: 85 \\
\mathrm{SiO}_{2}: 3200 \mathrm{H}_{2} \mathrm{O}\end{array}$} & \multirow{3}{*}{$\begin{array}{l}\text { Sodium } \\
\text { silicate }\end{array}$} & 160 & \multirow[t]{3}{*}{ TEPABr } & - & - & - & $2.5 \times 10-2$ & 4.9 & \multirow[t]{3}{*}{117.2} & \multirow[t]{3}{*}{-} & \multirow[t]{3}{*}{89.8} & \multirow[t]{3}{*}{6} \\
\hline & & 180 & & - & - & - & $6.1 \times 10-2$ & 3.5 & & & & \\
\hline & & 200 & & - & - & - & $15.1 \times 10-2$ & 1.8 & & & & \\
\hline \multirow{3}{*}{$\begin{array}{l}\text { 4.5(TPA })_{2} \mathrm{O}: 12 \mathrm{LiO}_{2}: \\
\mathrm{Al}_{2} \mathrm{O}_{3}: 90 \mathrm{SiO}_{2}: 200 \mathrm{H}_{2} \mathrm{O}\end{array}$} & \multirow[t]{3}{*}{ Ludox } & 140 & $\mathrm{TPABr}$ & - & - & - & - & - & 7.5 & & 22.5 & 7 \\
\hline & & 155 & & - & - & - & - & - & (kcal/ & & & \\
\hline & & 170 & & - & - & - & - & - & $\begin{array}{c}\text { g.mol } \\
. \mathrm{K})\end{array}$ & & & \\
\hline $4.5(\mathrm{TPA})_{2} \mathrm{O}: 12 \mathrm{~K}_{2} \mathrm{O}:$ & Ludox & 140 & $\mathrm{TPABr}$ & - & - & - & - & - & 15.0 & - & 23.5 & 7 \\
\hline $\mathrm{Al}_{2} \mathrm{O}_{3}: 90 \mathrm{SiO}_{2}: 200 \mathrm{H}_{2} \mathrm{O}$ & & 155 & & - & - & - & - & - & & & & \\
\hline & & 170 & & - & - & - & - & - & & & & \\
\hline $4.5(\mathrm{TPA})_{2} \mathrm{O}: 12 \mathrm{Na}_{2} \mathrm{O}$ & Ludox & 140 & TPABr & - & - & - & - & - & 85 & & 20 & 7 \\
\hline $\mathrm{Al}_{2} \mathrm{O}_{3}: 90 \mathrm{SiO}_{2}: 200 \mathrm{H}_{2} \mathrm{O}$ & & 150 & & - & - & - & - & - & & & & \\
\hline & & 160 & & - & - & - & - & - & & & & \\
\hline & & 170 & & - & - & - & - & - & & & & \\
\hline 4.5(TPA $)_{2} \mathrm{O}: 12 \mathrm{Na}_{2} \mathrm{O}:$ & Ludox & 150 & $\mathrm{TPABr}$ & - & - & - & - & - & 32 & & - & 7 \\
\hline $\mathrm{Al}_{2} \mathrm{O}_{3}: 70 \mathrm{SiO}_{2}: 200 \mathrm{H}_{2} \mathrm{O}$ & & 160 & & - & - & - & - & - & & & & \\
\hline & & 170 & & - & - & - & - & - & & & & \\
\hline
\end{tabular}




\section{References}

1. Corregidor, P. F., Acosta, D. E. \& Destéfanis, H. A. Kinetic Study of Seed-Assisted Crystallization of ZSM-5 Zeolite in an OSDA-Free System Using a Natural Aluminosilicate as Starting Source. Industrial and Engineering Chemistry Research 57, 13713-13720 (2018).

2. Pan, F. et al. Synthesis and crystallization kinetics of ZSM-5 without organic template from coal-series kaolinite. Microporous and Mesoporous Materials 184, 134-140 (2014).

3. Mintova, S., Valtchev, V., Vultcheva, E. \& Veleva, S. Crystallization kinetics of zeolite ZSM-5. Zeolites 12, 210-215 (1992).

4. Kim, S. D., Noh, S. H., Seong, K. H. \& Kim, W. J. Compositional and kinetic study on the rapid crystallization of ZSM-5 in the absence of organic template under stirring. Microporous and Mesoporous Materials 72, 185-192 (2004).

5. Kulkarni, S.B., Shilarkar, V.P., Kotasthane A.N. \& Borade, R.B. Studies in the Synthesis of ZSM-5 Zeolites, Zeolites 2, 313-318(1982)

6. Joshi, P.N., Rao, G.N., Kotasthane, A.N., Shilalkar, V.P. Influence of Template on Crystallization of ZSM-5 Zeolites, Journal of Inclusion Phenomena and molecular Recognition in Chemistry 9, 91-99 (1990)

7. Rysard, M. \& Sand, L.B., Crystallization of ZSM-5 with Relatively high ( $\left.\mathrm{Me}_{2 / \mathrm{n}}\right)_{2} \mathrm{O} /(\mathrm{TPA})_{2} \mathrm{O}$ Reactant Ratios Zeolites 2, 143-146 (1982) 